\title{
TERRESTRIAL FAUNA OF KASHIMA ISLETS IN THE BAY OF TANABE, WAKAYAMA PREFECTURE
}

$\operatorname{AUTHOR(S):~}$

Morikawa, Kuniyasu

\section{CITATION:}

Morikawa, Kuniyasu. TERRESTRIAL FAUNA OF KASHIMA ISLETS IN THE BAY OF TANABE, WAKAYAMA PREFECTURE. PUBLICATIONS OF THE SETO MARINE BIOLOGICAL LABORATORY 1957, 6(2): 225-240

ISSUE DATE:

1957-12-25

URL:

http://hdl.handle.net/2433/174578

RIGHT: 


\title{
TERRESTRIAL FAUNA OF KASHIMA ISLETS IN THE BAY OF TANABE, WAKAYAMA PREFECTURE ${ }^{13}$
}

\author{
KUNIYASU MORIKAWA
}

Biological Institute, Ehime University, Matsuyama

The Islets of Kashima are located in the Bay of Tanabe, and are about $1 \mathrm{~km}$ apart from the mainland, Kii Peninsula, and $3.5 \mathrm{~km}$ east of the Seto Marine Biological Laboratory at Shirahama, Wakayama Prefecture. The Islets are actually formed of two small islets, joined by natural bridge of rocks. These two islets are about $700 \mathrm{~m}$ and $300 \mathrm{~m}$ round respectively. Both of them are less than $20 \mathrm{~m}$ at the highest, and they are constituted of the Tertiary rocks. They are covered with primeval dense forests which are composed of Rhus silvestris, Ilex integra, Machilus Thunbergii, Daphniphyllum Teijsmannii, Camellia japonica, Cinnamomum japonicum, Prunus Zippeliana, etc. Owing to the abundant flora which contains some subtropical plants, the islets have been designated as a natural monument. The flora have been well surveyed and 160 species of higher plants have been recorded previously. But, hitherto few have been known of the terrestrial fauna except the land snails. This is the reason why the present investigation has been undertaken, and the writer had an opportunity to collect and investigate the land animals of these islets from $\mathbf{1}$ to $\mathbf{5}$ June, 1956, through the good offices of Dr. Huzio UTinomi of the Seto Marine Biological Laboratory.

Subterranean animals were collected from forest soil within the depth of about $2.5 \mathrm{~cm}$ by using Berlese funnels, and the soil was of so-called mull type and showed acidity of $\mathrm{pH} 5.8$ on an average. Drosophilids are caught by banana-trapping, and other animals were collected by searching the litter layers of the forest and the seashore drifts, and by peeling the bark of trees.

The collected animals were classified into 226 species, 193 genera, 123 families, and 31 orders belonging to 4 phyla, as shown in Table 1.

The findings of such subtropical and noticeable species as Tornatellides boeningi inexpectus, Philoscia muscorum, Kashimachelifer cinnamomeus, Otocryptops rubiginosus kasimensis, Duolandrevus coulonianus, Laius asahinai, etc. are of much interest from the zoogeographical view-point.

1) Contributions from the Seto Marine Biological Laboratory, No. 304.

Publ. Seto Mar. Biol. Lab., VI (2), 1957. (Article 14) 
Table 1.

\begin{tabular}{r|r|r|r|r|r}
\hline & Class & Order & Family & Genus & Species \\
\hline Annelida & 1 & 2 & 3 & 4 & 4 \\
\hline Mollusca & 1 & 1 & 8 & 13 & 14 \\
\hline Arthropoda & 6 & 25 & 108 & 172 & 204 \\
Crustacea & & 2 & 4 & 5 & 5 \\
Arachnida & 4 & 35 & 48 & 56 \\
Diplopoda & 3 & 3 & 3 & 3 \\
Symphyla & 1 & 2 & 2 & 2 \\
Chilopoda & & 3 & 6 & 8 & 9 \\
Insecta & & 12 & 58 & 106 & 129 \\
\hline Vertebrata & 2 & 3 & 4 & 4 & 4 \\
\hline Total & 10 & 31 & 123 & 193 & 226 \\
\hline
\end{tabular}

\section{Acknowledgments}

The collected animals were sent to specialists for identification, except the molluscs which were referred to the description of Dr. Tokubei KuRODA ${ }^{12}$ and such groups familiar to the writer as pseudoscorpions, mites, etc. Consequently, the writer is greatly indebted to many following persons who identified the present materials:

Dr. Hideji Yamaguchi, Hokkaido Gakugei University, Hakodate (Archioligochaeta); Dr. Shinryo OHfuchi, Tokyo Agricultural University (Neoligochaeta); Dr. Huzio Utinomi, Seto Marine Biological Laboratory, Shirahama (Crustacea); Mr. Takeo Yaginuma, Otemon Gakuin, Osaka (Araneina); Dr. Seisho Suzukr, Hiroshima University (Opiliones); Mr. Yasunori MiyosI, Matsuyama Kita High School (Millipedes); Dr. Ryozo Yosir, Kyoto University (Collembola); Dr. Mikio Kurosawa, Nihon Noyaku Produc. Co., Tokyo (Thysanoptera); Dr. Tamotsu IshinARA (Heteroptera and Orthoptera), Mr. Mutsuo Mryatake and Mr. Sadanari Hrsamatsu (Coleoptera), Mr. Susumu UEDA (Wasp), Mr. Fumihiko TAKechi (Formicidae in part), and Mr. Masayoshi OHUE (Collembola in part), Ehime University; Mr. Kei Oкамото, Heiwa Middle School, Kochi Prefecture (Formicidae); Mr. Katsura MorImoto, Kyushu University (Cur. culionidae in part); Dr. Toyohi OKadA, Tokyo Metropolitan University (Drosophila). Dr. Tadashige HABE of Kyushu University kindly inserted corrections regarding the scientific names of molluscs.

The writer wishes to express hearty thanks to Dr. H. Utinom, Mr. Torao YAMAMOTo and other members of the Seto Marine Biological Laboratory, Mr. Shin Goto of the Ena Middle School, Wakayama Pref., and the members of the protection committee of the natural monument of Tanabe City for their kind co-operations in the

1) Kuroda, T., Venus, $13(5 / 8): 313-316,1944$. 
course of the present survey. His thanks are also due to Dr. Takeo ITo of Ehime University who was kind enough to read the manuscript of this report.

\section{LIST OF THE LAND ANIMALS OF KASHIMA ISLETS ${ }^{1}$}

\section{Phylum ANNELIDA}

Class OLIGochaeta

Order Neoligochaeta

Family Megascolecidae

Pheretima servinus HATAI et OHFUCHI モリミミズ 2 exs

\section{Family Lumbricidae}

Bimastus sp. (similar to B. parvus, but smaller) フクロナンミミズの 1 種 3 exs Eisenia foetida (SAvigny) ンマミミズ 8 exs

Eisenia or Pheretima sp. (juvenile) 7 exs

\section{Order Archioligochaeta \\ Family Enchitraeidae}

Pachydrilus? sp. ヒメミミズの1種 about 300 exs

\section{Phylum MOLLUSCA \\ Class GASTROPODA \\ Order Stylommatophora \\ Family Elasmatidae}

Tornatellides boeningi inexpectus (PILSBRY) ノミガイ2)

Family Clausiliidae

Tyrannophaedusa plicilabris (A. ADAMS) コスジギセ abundant

Reinia variegata (A. ADAMS) ヒロクキコギセル common

Family Achatinidae

Allopeas pyrgula Schmacker et BoETTGER ホソオカチョウジ仿 abundant

Family Discidae

Punctum rota PILSBRy et Hirase クルマナタネ

1) Abbr. $a=$ adult, $s=$ subadult, $y=$ immature, deut=deutonymph, prot=protonymph, trit $=$ tritonymph.

2) Subtropical species, and distributed in S. Kyushu and Izu-hichi-to Islands. 


\section{Family Helixarionidae}

Parakaliella crenulata (GUDE) カ少キビ

P. habei (Kuroda) ハベキビ

Parasitara pallida (PILSBRY) ウスイロンダ

Nipponochlamys semisericata (?) (PILSBRY) キヌツヤベッコウ

Family Camaenidae

Satsuma japonica peculiaria (A. ADAMS) ムロマイマイ abundant

Family Bradybaenidae

Euhadra gulicki sigeonis KURODA シゲオマイマイ abundant

Bradybaena similaris (FÉRUSSAC) オナジイマイ abundant

Acusta sieboldiana (PFEIFFER) ウスカワマイマイ common

Family Streptaxidae

Guella (Sinoennea) iwakawa (PILSBRY) タワラガイ abundant

\section{Phylum ARTHROPODA \\ Class CRUSTACEA \\ Order Amphipoda \\ Family Talitridae}

Orchestia platensis KRÖYER ヒメ八マトビムシ $4 \hat{\jmath}, 15 q, 3 s \hat{b}, 14 s$ 市, $89 y$

\section{Order Isopoda}

Family Porcellionidae

Porcellio scaber LATREILle ワラシムシ 2o, $1 y$

\section{Family Oniscidae}

Alloniscus perconvexus (DANA) タマワラシムン 3 古

Philoscia muscorum (SCOPOLI) BUNDE-Lund カナエムレの1種1) $2 \hat{8}, 11$ 市

Family Armadillididae

Cubalis dorsalis (Iwamoto) セグロコンビロテマリムシ 99a,472y

\section{Class Arachnida}

Order Pseudoscorpionidea

Family Chthoniidae

Mundochthonius japonicus CHAMBERLIN メクラヒメツテカニムシ 29お, 12ᄋ, 1 trit Tyrannochthonius dogoensis MORIKAWA ムネトゲツチカニムン 1 今, 1 †

1) Recorded in Japan for the first time. 
Family Neobisiidae

Neobisium (Parobisium) sp. A. コケカニムシの 1 種 2 trit, 26 deut, 2 prot

Family Chernetidae

Toxochernes japonicus Morikawa モリケルネス 10\}, 21 우 7 trit, 26 deut, 3 prot

Family Cheliferidae

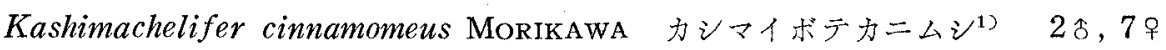

\section{Order Araneina}

Family Ctenizidae

Ummidia fragaria (DöNITZ) キノボリトタテグモ $1 y$ ㅇ

Family Segestriidae

Ariadna lateralis (KARSCH) ミヤグモ $1 s$ 市

Family Teridiidae

Teridion crassipes (BOESENBERg et STRAND) コノハヒメグモ 1 古

Th. sterninotatum BoEsENBERG et STRAND ムナボンヒメグモ $1 s$ 운

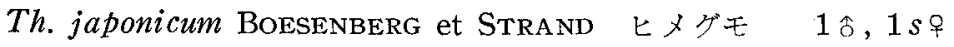

Th. sp. ヒメグモの 1 種 1 ex

Dipoena sp. ミシングモの 1 種 1 ex

Family Micryphantidae

Oedothorax sp. アカムネグモの1種 3 exs

Gen. spp. コサラグモ科の数種 abundant

Family Agriopidae

Neoscona subpullata (Boesenberg et STRAND) ヘリシロオニグモ2 2 ㅇ

N. mellotteei Simon ワキグロサッマノミダマン $1 y$ 市

Leucauge sp. ンロガホグモの1種 1 ex

Family Lycosidae

Pirata piratoides (BoESEnBERg et Strand) ヒナドクグモ 1 우

Lycosa spp. ドクグモの数種 abundant

Family Oxyopidae

Oxyopes sertatus L. КосH ササグモ $1 s$ ㅇ

1) Found under the bark of tree (Cinnamomum japonicum), and described in Zool. Mag. (Jap.) 66 (10) : 399-402, 1957. 
Family Agelenidae

Agelena sp. クサグモの 1 種 1 ex

Caelothes sp. ヤチグモの 1 種 1 ex

Tegenaria sp. タナグモの1種. 1 ex

\section{Family Thomisidae \\ Xysticus ephippiatus SIMON ヤミイロカニグモ $2 \hat{\delta}$ \\ Family Salticidae}

Myrmarachne japonica (KARSCH) アカアリグモ $1 s$ ㅇ

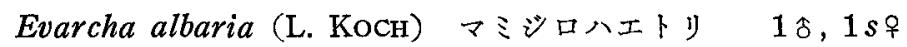

\section{Family Clubionidae}

Itatsina praticola (BoESENBERG et STRAND) イタチグモ $1 s$ 古, many $y$ 우

Clubiona sp. フクログモの 1 種 1 ex

Family Heteropodidae

Heteropoda forcipata (KARSCH) コアンダカグモ $1 s$ ㅇ, $2 \boldsymbol{y}$ ㅇ

Family Ctenidae

Anahita fauna KARSCH レボグモ 1 우 $1 s$

\section{Order Opiliones}

Family Phalangodidae

Pseudobiantes japonicus Hirst カマキリザトウムン 6 exs 〔lives in the dark place (troglophilous animal)]

Family Phalangiidae

Gagrella japonica RoEwER ヒトハリザトウムン 5 exs (maritime animal)

\section{Order Acarina}

Family Macrochelidae

Macrocheles sp. 1 マエツメナンダニの1種１4名,22우

M. sp. 210 우

M. sp. 31 우

Macrochelidae? sp. $18 \hat{\jmath}, 98$ ㅇ, $141 y$

Family Gamasolaelaptidae

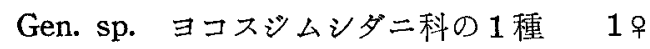


Family Parasitidae

Parasitus sp. キセイダニの 1 種 $10 \hat{0}, 20$ 早

Family Neoparasitidae

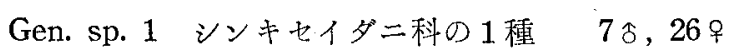

Gen. sp. $2 \quad 1$ ㅇ

Family Calyptostomidae

Gen. ? sp. サヤアゴダニ科の 1 種 ? 1 中

Family Cunaxidae

Cunaxas sp. ヒメテングダニの1種 $1 s$ 9

Family Labidostomidae

Labidostoma sp. ヨロイダニの1種 2 ㅇ

Family Acaridae

Rhizoglyphus? sp. ホダニの1種？ 4 exs

Family Nanhermanniidae

Nanhermannia sp. pc フクロ八ラヤマダニ $1 e x$

Family Epilohmanniidae

Epilohmannia sp. ja ヨコミゾヤマダニ 1 ex

Family Lohmanniidae

Torpacarus sp. ka ッッハラヤマダニ $106 a, 116 y$

Family Hypochthoniidae

Gen.? sp. チカノヤマダニ科の 1 種 1 ex

Family Camisiidae

Gen. (closely akin to Heminothrus) sp. 七クボヤマダニ科の 1 種 1 ex Nothrus sp. pb セクポヤマダニ 5 exs

Family Hermanniidae

Gen.? sp. ナカダカヤマダ二科の 1 種 3 exs

Family Eremaeidae

Eremaeus sp. ke ノロモリダニ 1 ex

Family Phthyracaridae

Phthyracarus sp. hz ハコガメダニ $12 a, 11 y$ 
Oribotritia sp. pd ミツズメハコガメダニ 8 exs $O . ?$ sp. hh 2 exs

Pseudotritia sp. sh ホソハコガメダニ 1 ex

\section{Class Diplopoda}

Order Polyxenomorpha

Family Polyxenidae

Monographis takakuwai MryosI ハイボクフサヤスデ10 exs (abundant on the ground and trees)

Order Polydesmoidea

Family Strongylosomatidae

Ndyopus patrioticus patrioticus (ATTEMS) ヤマトアカヤスデ２各，6오

\section{Order Juliformia}

Family Julidae

Fusiulus sp. ヒメャスデの1種 2 ๖, $5 y$

\section{Class SymphyLA}

Order Symphyla

Family Scolopendrellidae

Symphylella vulgaris HANSEN ヤサコムカデ 22 exs

Family Scutigerellidae

Hanseniella sp.? ナミコムカデ 1 ex

\section{Class ChILOPODA}

Order Scolopendromorpha

Family Scolopendridae

Scolopendra subspinipes japonica L. KocH アオズムカデ 4 exs

Family Cryptopidae

Otocryptops rubiginosus (L. KocH) セスシアカムカデ 1 ex

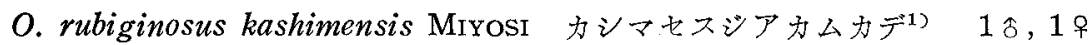

1) Newly found and described by Y. MiYoshI in Zool. Mag. (Jap.) 66 (6) : 256-257, 1959. 
Order Geophilomorpha

Family Schendylidae

Momophilus serratus TAKAKUWA そモジムカデ 2 exs

Thalthybius tenuicolis TAKAKUWA サキブトジムカデ 3 exs

Family Mecistocephalidae

Mecistocephalus fenestratus VERHOEFF マドナガズシムカデ 5 exs

M. marmoratus VERHOEFF ブチナガズシムカデ５人，9ᄋ

Family Geophilidae

Pleurogeophilus sp. ツチムカデの 1 種 $12 y$

Order Lithobiomorpha

Family Lithobiidae

Bothropolys imaharensis VERHOEFF イマハルイッスンムカデ 15 exs

Monotarsobius ramulosus TAKAKUWA フンザンヒトフンムカデ 12 exs

Class Insecta

Order Thysanura

Family Japygidae

Japyx japonicus ENDERLINE ヤマトハサミコムシ 7 exs

Order Collembola

Family Hy pogastruridae

Hypogastrura communis exillis YosII ムラサキトビムシ $1 y$

Family Neanuridae

Neanura (Propeanura) mandarina Yosı オオアカイポトビムン $2 y$

N. sp. $1 \boldsymbol{y}$

Metanura sancti-sebastiani YosII 1 a

Family Brachystomellidae

Odonthella sp. $\quad 2 y$

\section{Family Onychiuridae}

Onychiurus yagii MryosHI ヤギトビムンモドキ $8 a$

O. flavescens YosII $5 a, 4 y$

O. spp. (containing O. uenoi YosII?) $82 y$ 
Family Isotomidae

Folsomia fimetaria LINNÉ? フォルソムトビムン $3 a$ Isotoma trispinata MACGILlIVRAY ミドリトビムン $4 a$ I. sp. $\quad 83 y$ Isotomiella minor (SCHAFFERS) $1 y$

Family Achorutidae

Pseudoachorutes sp. $\quad 3 a$

Family Entomobryidae

Sinella umesaoi Yosir? $1 y$

Homidia sauteri BöRNER ザウテルアヤトビムン， $7 a, 7 y$

\section{Order Orthoptera}

Family Gryllidae

Duolandrevus coulonianus DE SAUSSURE クチキコオロギ $4 y$ (subtropical species)

Family Mogoplistidae

Ornebius bimaculatus SHIRAKI イソカネタタ $2 s$ 古, $2 y$ (subtropical species)

Family Phyllodromiidae

Phyllodromyia lituricollis WALKER モリチャバホゴキブリ 1 \&ิ, 2 古, $2 y$

Ph. kumamotonis SHIRAKI スカンバチビゴキブリ $1 \hat{\jmath}, 3 y$

Order Dermaptera

Family Anisolabiidae

Anisolabis maritima BORELLI ハサミムシ $14 y$

Order Isoptera

Family Termitidae

Coptotermes formosanus ShIRAKI イエンロアリ 52

Order Thysanoptera

Family Thripidae

Hoplothrips flavipes (BAGNALL) クチキクダアザミウマ 3 exs

Order Hemiptera

Family Cicadidae

Terpnosia vacua OLIVIER ハルゼミ abundant 
Family Miridae

Deraeocoris rufus Distant 1 ex

Family Reduviidae

Sphedanolestes impressicollis STÅL レマサンガメ 1 ex

Family Coreidae

Hygia opaca UHLER ツマキーリカメムン 8 exs

Family Pentatomidae

Megymenum gracilicorne DALLAS ノコギリカメムン 1 ex Glaucias subpunctatus WALKER ツヤアオカメムシ 1 ex Nezara antenuata ScotT アオクサカメムシ 1 ex

Family Thyreocoridae

Adrisa magna (UHLER) $\exists コ ス ゙ ナ ツ チ カ メ ム 2^{11}$

\section{Order Neuroptera \\ Family Chrysopidae}

Chrysopa sp. クサカゲロウの 1 種 1 ex

\section{Order Lepidoptera}

Family Geometridae

Archanna jaguararia GUÉNÉE ヒョウモンエダンャタ 3 exs

Family Pieridae

Eurema hecabe LINNÉ キチョウ 5 exs

Pieris rapae crucivora BoISDUvaL モンンロテョウ 6 exs

Family Papilionidae

Graphium sarpedon nipponus FRUHSTORFER アオスジアグ八 2 exs

Family Satyridae

Ypthima argus BUTLER ヒメウラナミシャノメ 2 exs

Order Coleoptera

Family Paussidae

Eustra plagiata japonica BATES エグリゴミムン 3 exs

1) After Goto, S., Nanki-Seibutsu II (2) : 95, 1950. 


\section{Family Harpalidae}

Tachys sericans BATES ウスオビコミズギワゴミムシ 2 exs

Platymetopus flavilabris corrosus BATEs 1 ex

Calathus (Crepidactyla) nitidus Motschulsky オオクロツヤヒラタゴミムン 3 exs

C. (Pristodactyla) dulcigradus BATES ヒメッヤヒラタゴミムン 1 ex

Family Staphylinidae

Osorius taurus SHARP ツノフトッッハネカクン 2 exs

Domene crassicornis SHARP オオマルズハホカクン 2 exs

Lathrobium partitum SHARP サキアカバナガ八ネカクン 3 exs

Philonthus rectangulus SHARP カクコガンラハネカクン 7 exs

$P h$. sp. カクコガンラハネカクンの 1 種 1 ex

Cafius vestitus SHARP アバタウミベハホカクン 1 ex

Phucobius simulator SHARP ウミベアカバハネカクン 15 exs

Ischnopoda (Microdota) sp. 1 ex

Thamiaraea japonica CAMERON 3 exs

Amarochara (Amarochara) sp. 1 ex

Family Hydrophilidae

Cercyon sharpi HAROLD フチトリケンガムシの1種４exs

Megasternum gibbulum Motschulsky マグソガムシの1種 2 exs

Family Lampyridae

Psilocladus ruficollis KIESENwetTeR ムネクリイロポタル 1 ex

Family Dasytidae

Laius asahinai NAKANE イソジョウカイモドキ 1 ex (interesting species and live in the maritime drifts)

Family Nitidulidae

Haptoncus ocularis FAIRMAIRE ケンキスイの1種 5 exs

Family Coccinelidae

Epilachna sparsa orientalis DIEKE ニジュウヤホンテントウ 1 ex

Hyperaspis japonica CROTCH ウスフタホンデトウ 1 ex

Scymnus (Pullus) hilaris Motschulsky 2 exs

Serangium japonicum CHAPIN 1 ex

Amida tricolor HAROLD アミダテントウ 2 exs

Family Elateridae

Dicronychus (Platynchus) adjutor CANDEZE アカアンハナコメッキ 1 ex 


\section{Family Dermestidae}

Trinodes rufescens REITTER キビケカツオブンムン 1 ex

Gastrallus laevigatus OLIvier 1 ex

\section{Family Ciidae}

Cis (Hadraule) bifasciatus REITTER フタオビツッキノコムシ 2 exs

Family Sphindidae

Aspidiphorus sp. ヒメキノコムンの1種 1 ex

Family Tenebrionidae

Idisia ornata PASCOE ハマヒョウタンゴミムンダマン 1 ex

Micropedinus algae LEWIS ホソハマベゴミムシダマシ 23 exs

M. pallidipennis LEWIS ヒメホソハマベゴミムンダマン 1 ex

Family Alleculidae

Hymenaria rufipennis MARSEUL アカハネヒメクチキムン 1 ex

Family Curculionidae

Trachyphloeosoma sp. A 1 ex

T. sp. B 1 ex

Curculio funebris Roelofs 2 exs

Myosides seriehispidus RoELOFS テビヒョウタンゾウムン 8 exs

Phyllobius brevitarsis KoNo コヒゲボソゾウムン 1 ex

Stenoscelis gracilitarsis WOLLASTON 5 exs

Demimaea circula RoELOFS マルモンササラゾウムン 2 exs

Cryptorrhynchidius insidiosus RoELOFs マツシラホンゾウムシ 3 exs

Acalles sp. $\quad 8$ exs

Gen. sp. (Gen. \& sp. nov. belongings to new Tribe) メクラッヤゾウムン 10 exs

Family Ipidae (Scolytidae)

Xyleborus rubicollis EICHHOFF アカクビキクイムン 1 ex

Cryphalus sp. コキクイムシの1種 2 exs

Family Trogidae

Trox opacotuberculatus Motschulsky ヒメコブスシコガネ 1 ex

Family Scarabaeidae

Panelus parvulus WATERhouse マメガムンガタコガネ 1 ex

Cetonia pilifera MotSChULSKy ハナムグリ 1 ex 


\section{Order Hymenoptera}

Family Ichneumonidae

Diplozon laetatorius (FABRICIUS) アカハラララタアブヤドリバテ 1 ex

Family Vespidae

Polistes japonicus fadwigae DALlA TORRE セグロアンナガバチ Vespa mandarina SMITH スズメバキ 3 exs

\section{Family Crabronidae}

Lestica $(=$ Crabro $)$ sp. ギンクチバチの 1 種 1 ex

Family Halictidae

Halictus sp. コハナパキの1種 4 exs

Family Formicidae

Amblyopone silvestrii WHEELER ノコギリハリアリ 4 Ð

Sysphincta watasei WHEELER ワタセバリリ $10 \nvdash$

S. sp. $1 \Varangle$

Ponera scabra WHEELER テラニシハリアリ 5 市

$P$. sp. $1 \Varangle$

Pheidole pieli SantschI オオズアカアリの1種１32f, 98

Crematogaster laboriosa SMITH? トビイロシリアダアリ $3 \Varangle$

C. sordidula osakensis FOREL キイロンリアゲアリ 147 氒

Myrmecina graminicola nipponica WHEELER カトフンアリ $7 \Varangle$

Pristomyrmex pungens MAYER アミメアリ $3 \not{\gamma}$

Olygomyrmex sauteri FoREL コッノアリ $42 f, 37 \Varangle$

Strumigenis lewisi CAMERON ウロコアリ 1 ㅇ, $6 \Varangle$

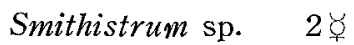

Polyrhachis hippomanes moesta EMERY チクントグアリ 1 \&

Paratrechina flavipes SMITH アメイロ

Lasius niger LINNÉ? トビイロケアリ10

Formica fusca japonica MotschuLsKy クロヤマアリ 51 文

Camponotus (Camponotus) sp. オオアリの1種 $1 \Varangle$

\section{Order Diptera}

\section{Family Culicidae}

Aedes albopictus Skuse ヒトスジンカ abundant

A. niveus LudLOW ンロカタヤブカ common

A. togoi Theobald トウゴウヤブカ common 
Family Tipulidae

Gen. sp. ガガンボ科の1種 1 ex

Family Chironomidae

Chironomus (Chironomus) sp. 1 ex

Family Dolichopodidae

Psilopus nebulosus Matsumura? マダタアンナガバエ 2 exs

Family Syrphidae

Epistrophe balteatus DE GEER? ホソヒラタフブ 1 ex

Family Drosophilidae

Drosophila (Sophophora) auraria PENG, type A カオシロショウジョウパエA型 $8 \delta, 2$ 方

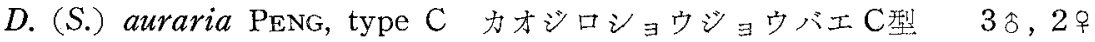

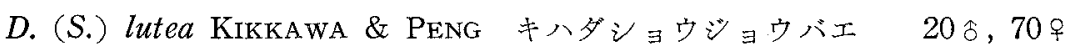

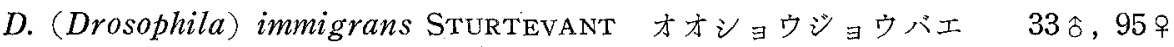

D. (D.) bizonata Kikkawa \& Peng フタオビンョウショウパエ 1〕, 1 市

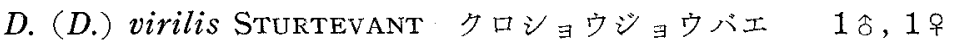

D. (D.) sordidula KIKKAwa \& PENG オオクロンョウショウパエ $1 \hat{\jmath}$

D. (D.) sternopleuralis OKADA $1 \hat{\jmath}$

Family Muscidae

Musca hervei VILLENEUve イエパエ 1 웅

Muscina stabulans (FALLEN) オオイエパエ $1 \hat{o}, 1$ ex

Ophyra leucostoma (WIEDEMANN) ヒメクロパエ 1 市

Family Anthomiidae

Gen. sp. 八ナパ工科の1種 1 古

Family Calliphoridae

Lucilia ampullacea (VILleneuve) コ价ホキンハエ $1 \delta, 3$ 市

L. sp. 1 ㅇ

Family Sarcophagidae

Sarcophaga melanura MeIGEN シリグロニクバエ 2 우

Phylum VERTEBRATA

Class Reptilia

Order Lacertilia

Family Lacertidae

Takydromus tachydromoides (SCHLEGEL) カナへビ abundant 


\section{Class Aves}

Order Columbae

Family Columbidae

Streptopelia orientalis orientalis (LATHAN) キジバト

\section{Order Passera}

Family Turdidae

Monticola solitarius magnus (LA Touche) イソヒヨドリ

\section{Family Corvidae}

Corvus levaillantii japonensis BoNAPARTE ハシブトガラス 